\title{
Review
}

\section{A RING to rule them all? Insights into the Map3k1 PHD motif provide a new mechanistic understanding into the diverse roles of Map3k1}

\author{
T Suddason ${ }^{* 1}$ and E Gallagher*1
}

Despite the sizable number of components that comprise Mapk cascades, Map3k1 is the only element that contains both a kinase domain and a plant homeodomain (PHD) motif, allowing Map3k1 to regulate the protein phosphorylation and ubiquitin proteasome systems. As such, Map3k1 has complex roles in the regulation of cell death, survival, migration and differentiation. Numerous mouse and human genetic analyses have demonstrated that Map3k1 is of critical importance for the immune system, cardiac tissue, testis, wound healing, tumorigenesis and cancer. Recent gene knockin of Map3k1 to mutate the E2 binding site within the Map3k1 PHD motif and high throughput ubiquitin protein array screening for Map3k1 PHD motif substrates provide critical novel insights into Map3k1 PHD motif signal transduction and bring a brand-new understanding to Map3k1 signaling in mammalian biology.

Cell Death and Differentiation (2015) 22, 540-548; doi:10.1038/cdd.2014.239; published online 23 January 2015

Facts

- Of the 19 Map3ks only Map3k1 contains a plant homeodomain (PHD) motif, and is an E3 ubiquitin (Ub) ligase.

- The Map3k1 PHD motif regulates both Mapk cascade protein stability following hyperosmotic stress and Mapk pathway activation from transforming growth factor- $\beta$ (Tgf- $\beta$ ) and epidermal growth factor (Egf) cytokine receptors by the Ub-proteasome system.

- The Map3k1 PHD motif is critical for stem cell differentiation, tumorigenesis, B-cell development, T-cell signaling, protecting cardiac tissue from damage and testis development.

\section{Open Questions}

- Are divergent roles for the Map3k1 kinase domain and PHD motifs present within human breast cancers?

- Does the Map3k1 PHD motif regulate Tabs by noncanonical ubiquitination following CD40, and other Tnfrs, signal transduction?
- Are novel Map3k1 PHD motif substrates targeted by the Ubproteasome system during apoptosis?

\section{Discovery and Early Characterization}

Mitogen-activated protein kinase (Mapk) kinase (Map2k) kinases (Map3ks) activate Mapks by the binding and phosphorylation of Map2ks (Figure 1) $\cdot^{1-4}$ Map3k1 (encoded by Map3k1, and also known as MEKK1) was initially partially cloned as a complimentary DNA (cDNA) that encoded the C-terminal 672 amino acid residues of the Map3k and contains the kinase domain. ${ }^{5}$ Notably, the Map3k1 kinase domain shows a significant sequence homology to the Schizosaccharomyces pombe kinase Byr2 and the Saccharomyces cerevisiae kinase Ste11, both Map3ks of the yeast pheromone response pathway. ${ }^{5,6}$ But, despite its relatively high sequence similarity, Map3k1 cannot replace the function of Ste11 in yeast. ${ }^{6}$ Map3k1 is a serine and threonine kinase and a phospho-protein that was the second mammalian Map3k, after c-Raf, demonstrated to phosphorylate Map2k1 (also known as MEK1) within its activation domain. ${ }^{7-9}$ Subsequently, Map3k1 was shown to bind and activate Map2k4 (also known as MKK4 or JNKK1) that, in turn, phosphorylates the

${ }^{1}$ Department of Medicine, Imperial College London, Du Cane Road, London, UK

*Corresponding author: E Gallagher, Medicine, Imperial College London, Du Cane Road, London W12 ONN, UK, Tel: +44 7947 778996; Fax: +44 2075 640814; E-mail: ewen.gallagher@ntlworld.com or t.suddason@imperial.ac.uk

Abbreviations: Anf, Atrial natriuretic factor; Bcl-2, B-cell lymphoma 2; c-lap1/2, Cellular inhibitor of apoptosis proteins 1 and 2; Caspase, Cysteine-aspartic acid protease; DUBs, deubiquitinating enzymes; Egf, Epidermal growth factor; Egfrs, Egf receptors; ES, embryonic stem; Fak, Focal adhesion kinase; C-Flip, FLICE (FADD-like IL-1 $\beta$ converting enzyme)-inhibitory protein; HECT, Homologous to the E6-AP Carboxyl Terminus; Ikks, IkB $\alpha$ kinases; LPA, lysophosphatidic acid; MDCK, Madin-Darby canine kidney; MMTV LTR, mammary tumor virus long terminal repeat; Map2k, Mapk kinase; Map3k, Map2k kinase; Mapk, Mitogen-activated protein kinase; MEF, mouse embryonic fibroblast; NF- $\kappa$ B, Nuclear factor $\kappa$-light-chain-enhancer of activated B cells; PHD, plant homeodomain; PyMT, Polyoma Virus middle T antigen; PRR, Prorich region; RING, Really Interesting New Gene; Stam1, Signal transducing adapter molecule 1; SWIM, SWI2/SNF2 and MuDR; siRNA, Small interfering RNA; Tab, Tgf- $\beta$ activated kinase 1-binding protein; Tgf $\beta$ rs, Tgf- $\beta$ receptors; Th, T helper; Tnip, TNFAIP3 interacting protein; Tnfr, Tnf receptor; Traf, Tnf receptor-associated factor; Tgf- $\beta$, Transforming growth factor- $\beta$; Tnf, Tumor necrosis factor; Ube2N, Ub-conjugating enzyme E2 N; UIMs, Ub-interacting motifs; Ub, Ubiquitin; Upa, urokinase-type Plasminogen Activator; Ube2D, Ub-conjugating enzyme E2D; Ube2V1, Ub-conjugating enzyme E2 variant 1; Usp, Ub-specific protease; ZnF, zinc finger

Received 12.10.14; revised 15.12.14; accepted 17.12.14; Edited by G Melino; published online 23.1.15 


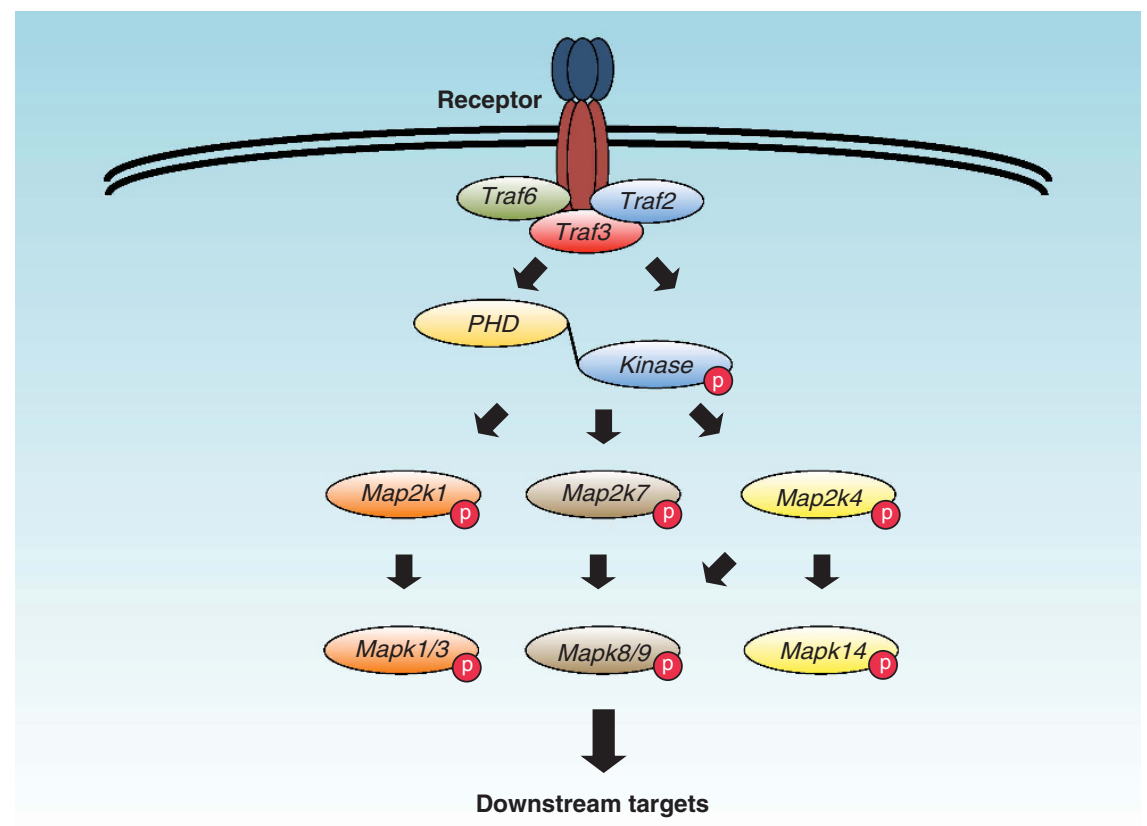

Figure 1 Schematic illustration of our early understanding of Map3k1 signal transduction. The Map3k1 kinase domain can phosphorylate and activate Map2ks that in turn phosphorylate and activate Mapks. Activated Mapk then phosphorylates downstream signaling targets in cells.

c-Jun N-terminal kinase (JNK) Mapk8 (also known as JNK1), Mapk9 (also known as JNK2) and Mapk14 (also known as p38-a). ${ }^{10-13}$ Early work showed that both Tumor necrosis factor (Tnf) $-a^{14}$ and crosslinking of the Tnf receptor (Tnfr) family member CD40 with antibodies activate Map3k1 in cell lines. ${ }^{15}$

The later cloning of the full-length rat Map3k1 cDNA revealed that Map3k1 possessed, in addition to its Ste11-like C-terminal kinase domain, a large $\mathrm{N}$-terminal regulatory region. ${ }^{16}$ Overexpression of full-length Map3k1 activates Mapk1/3, Mapk8/9 and Mapk14 pathways in fibroblast cell lines. ${ }^{1,16}$ Transfection of Map3k1 encoding cDNA into NIH3T3 cells leads to the activation of the nuclear factor $K$-light-chainenhancer of activated B-cell (NF-kB) pathway. ${ }^{17}$ Under these circumstances Map3k1 can form a complex with and induce the phosphorylation of $I_{k} \mathrm{~B} a$ kinases (Ikks) to activate the $\mathrm{NF}-\kappa \mathrm{B}$ pathway in cell lines. ${ }^{18}$ By similar bioinformatics methods that identified the Map3k1 kinase domain, other functional protein motifs have been identified within Map3k1 through computer sequence alignment techniques, including the PHD, ${ }^{19} \mathrm{Ub}$ interacting motifs ${ }^{19}$ and SWI2/SNF2 and MuDR domain. ${ }^{20}$ A multitude of Map3k1 binding partners have been found to date by a wide variety of molecular approaches (Table 1).

\section{Is Map3k1 a Mediator of Cell Death, Survival or Both?}

Map3k1 was initially suggested to be a pro-apoptotic kinase after several failed attempts to create stable cell lines that overexpress this Map3k. ${ }^{21}$ Indeed, inducible expression of the Map3k1 kinase domain can sensitize Swiss 3T3 cells to UV-irradiation-induced cell death. ${ }^{21}$ Similarly, inducible expression of the Map3k1 kinase domain in L929 fibrosarcoma cells can increase their susceptibility to Tnf- $a$-induced
Table 1 Listing of Map3k1 binding partners

\begin{tabular}{|c|c|c|}
\hline Protein & Function & Reference \\
\hline Nck interacting kinase & Kinase & 85 \\
\hline $14-3-3$ & Scaffold & 86 \\
\hline Mapk9 & Kinase & 87 \\
\hline a-actinin & Microfilament protein & 41 \\
\hline c-Raf & Kinase & 88 \\
\hline Mapk1 & Kinase & 88 \\
\hline RhoA & Small GTPase & 89 \\
\hline Cdc42 & Small GTPase & 90 \\
\hline Ras & Small GTPase & 91 \\
\hline Rac & Small GTPase & 90 \\
\hline p115 RhoGAP & GTPase-activating protein & 92 \\
\hline Map2k4 & Kinase & 12 \\
\hline Map2k7 & Kinase & 12 \\
\hline Map2k1 & Kinase & 5 \\
\hline C-Jun & Transcription factor & 32 \\
\hline Itch & HECT E3 Ub ligase & 51 \\
\hline Traf2 & Scaffold & 93 \\
\hline Grb2 & Adapter & 94 \\
\hline Axin & Scaffold & 95 \\
\hline Fak & Kinase & 96 \\
\hline Deltex 1 & E3 Ub ligase & 73 \\
\hline lkka & Kinase & 18 \\
\hline $\mathrm{lkk} \beta$ & Kinase & 18 \\
\hline Ikk $\gamma$ & Scaffold & 53 \\
\hline Tax & Nuclear factor & 97 \\
\hline Han11 & Scaffold & 98 \\
\hline MarvelD3 & Tight junction protein & 37 \\
\hline Tab1 & Scaffold & 72 \\
\hline
\end{tabular}

apoptosis. $^{22}$ Sequence analysis of full-length Map3k1 identified a short Cysteine-aspartic acid protease (Caspase) -3 cleavage site $\left({ }^{871}\right.$ DTVD $\left.^{874}\right)$, and its mutagenesis can prevent Map3k1-induced apoptosis caused by the overexpression of full-length Map3k1 encoding cDNA in cells. ${ }^{22}$ Caspase-3 cleavage at this site generates two Map3k1 fragments, the 
N-terminal fragment containing the PHD motif and the C-terminal fragment containing the kinase domain., ${ }^{5,19}$ Expression of Map3k1 with a mutant Capase-3 cleavage site can also prevent Map3k1 cleavage in response to genotoxic stress in fibroblast cell lines and following CD95 (also known as Fas) -mediated apoptosis in the Jurkat T-cell line. ${ }^{23,24}$ Anoikis, apoptosis induced by cellular detachment from extracellular matrices, can also initiate Map3k1 cleavage by Caspase-3, and an inactive kinase domain form of Map3k1 can inhibit anoikis-induced programmed cell death upon overexpression in Madin-Darby canine kidney cells. ${ }^{25}$ By contrast, Map3k1 is not susceptible to Caspase-3 cleavage following Madin-Darby canine kidney cell death induced by microtubule disruption drug treatment, ${ }^{26}$ demonstrating that Caspase-3 cleavage is not the only mechanism of Map3k1mediated cell death. Expression of the anti-apoptotic protein B-cell lymphoma 2 can block Map3k1-mediated apoptosis, though B-cell lymphoma 2 overexpression by itself is insufficient to prevent the Map3k1 cleavage. ${ }^{26}$ Overexpression of Map3k1 initiates a substantial Mapk8/9 activation in many cell types and is the likely mechanism for the proapoptotic role of the Map3k1 kinase domain. ${ }^{10,13}$ However, the induction of apoptosis by Mapk8/9 is a complicated process in cells and numerous mechanisms have been proposed to account for it, including activation of Itch by Mapk8 phosphorylation and Lys48-linked poly-Ub transfer onto cellular FLICE (FADD-like IL-1 $\beta$-converting enzyme)-inhibitory protein (cFlip) leading to c-Flip proteasomal degradation and the promotion of apoptosis, ${ }^{27}$ the control of p53 stability and cell death by Mapk8/9 phosphorylation of p53 at Ser6, ${ }^{28}$ and Mapk8/9 phosphorylation and regulation of B-cell lymphoma 2 family members to promote cell death. ${ }^{4}$

While the early analysis of Map3k1 by transfection and overexpression of its cDNA into cell lines suggested a proapoptotic role, these initial results in cell lines were then complicated by the contradictory finding that null mutant Map3k1 (Map3 $k 1^{-1-}$ ) mouse embryonic stem (ES) cells display enhanced cell death in response to hyperosmotic stress, low temperature shock and microtubule disruption drug treatment. ${ }^{29,30}$ These important results demonstrated that Map3k1 has a more critical role in protecting mammalian cells from many types of cell death, and that the role of Map3k1 in promoting apoptosis may well be a labyrinthine one. Similarly, Map3 $k 1^{-1-}$ ES cell-derived cardiac myocytes show enhanced cell death in response to oxidative stress. ${ }^{31}$ Most likely Map3k1-dependent Mapk activation reduces cell death by the activation of pro-survival targets. ${ }^{1,3}$

In addition to the Map3k1 kinase domain, roles for the Map3k1 PHD motif in cell death have been described. ${ }^{19,32,33}$ The Map3k1 PHD can mediate the transfer of Lys48-linked poly-Ub onto Mapk1, leading to the subsequent proteasomal degradation of Mapk1 in cell lines undergoing hyperosmotic stress-induced apoptosis. ${ }^{19,34}$ Similarly, the Map3k1 PHD motif has been reported to mediate the transfer of Lys48linked poly-Ub onto the c-Jun transcription factor to promote its degradation by the proteasome in Map3 $\mathrm{K}^{-/-}$mouse embryonic fibroblast (MEF) cells undergoing hyperosmotic stressinduced apoptosis. $^{32}$ The Map3k1 PHD may also act as E3 Ub ligase for c-Jun in cells undergoing cisplatin-induced apoptosis. ${ }^{35}$ Both the Map3k1 PHD and kinase domains are essential for microtubule disruption drug-induced Mapk8/9 activation and apoptosis in Map3k1 $1^{-1-}$ DT40 cells. ${ }^{36}$

Recently, MarvelD3, a transmembrane component of tight junctions that is required for epithelial monolayer integrity during hyperosmotic stress, has been identified as a protein that forms a complex with Map3k1 in cells. ${ }^{37}$ MarvelD3 can relocalize Map3k1 in response to hyperosmotic stress and by this means can regulate Mapk8/9 activation. ${ }^{37}$ MarvelD3mediated attenuation of Map3k1 signaling is critical for epithelial cell survival while undergoing hyperosmotic stress. $^{37}$

\section{Cell Migration and Wound Healing}

The generation of kinase-deficient Map3k1 (encoded by Map3 $k 1^{\Delta K D}$ ) expressing ES cells revealed that Map3k1 is critical for both serum- and lysophosphatidic acid (LPA) -induced Mapk8/9 phosphorylation. ${ }^{38}$ Map3k $1^{\triangle K D}$ ES cells also display reduced serum-induced migration in the Boyden chamber chemotaxis assays. ${ }^{38}$ Epidermal keratinocytes extracted from Map3k $1^{\Delta K D}$ mice have defective Tgf- $\beta$, Activin A- and Activin B-induced migration in cell culture plate-based wound healing assays. ${ }^{2,39}$ Map $3 k 1^{\Delta K D}$ keratinocytes display reduced Mapk8/9 phosphorylation following treatment with Tgf- $\beta$, Activin A or Activin B. ${ }^{39}$ The molecular basis underpinning defective Map3k1-dependent migration during wound healing may be the reduced formation of actin stress fibers in Activin B-treated Map3k $1^{\Delta K D}$ keratinocytes. $^{39}$ The formation of Activin B-induced actin stress fibers in keratinocytes is dependent upon Mapk8/9 activity because they can be ablated by the pre-treatment of keratinocytes with the SP600125 inhibitor compound. 2,39

Map3 $k 1^{-1-}$ MEF cells are defective in their adherence to cell culture plates when centrifuged at low speed. ${ }^{40}$ Like

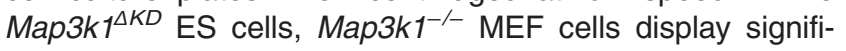
cantly reduced migration toward serum in the transwell migration assays. ${ }^{38,40}$ Similarly, migration toward fibronectin or fibronectin and Egf is reduced in Map3k $1^{-/}$MEF cells. ${ }^{40}$ Map3k1 has been shown by two groups to localizes to focal adhesions in fibroblasts, ${ }^{40,41}$ and less Vinculin, a critical cytoskeletal protein found in focal adhesions, is detected at the focal adhesions of Map3 $k 1^{-/}$MEF cells. ${ }^{40}$ Egf treatment of MEF cells leads to the formation of a complex between focal adhesion kinase (Fak) and Map3k $1 .{ }^{40}$ Map3 $\mathrm{k}^{-1-}$ MEF cells also display both reduced Mapk1/3 phosphorylation in response to Egf or Fibroblast growth factor-2 treatment and decreased Calpain activation, a calcium-dependent cysteine protease that is activated by Mapk $1 / 3$ phosphorylation. ${ }^{40,42}$

\section{Lymphocyte Differentiation and Effector Responses}

Naïve $\mathrm{CD}^{+} \mathrm{T}$ cells purified from the secondary lymphoid tissues of Map3k $1^{\triangle K D}$ mice and cultured under T helper (Th) 2 polarizing conditions secrete enhanced levels of Interleukins 4, 5, 10 and $13 .{ }^{43}$ By contrast, Th1 differentiation proceeds normally for $C D 4^{+} \mathrm{T}$ cells isolated from Map3k $1^{\Delta K D}$ mice. ${ }^{43}$ The aberrant Th2 phenotype identified in $\mathrm{CD}^{+} \mathrm{T}$ cells derived from Map3k $1^{\triangle K D}$ mice resembles the overproduction of Th2 cytokines found in Itchy mice, that harbor a promoter rearrangement mutation that ablates the expression of 
homologous to the E6-AP carboxyl terminus (HECT) E3 Ub ligase Itch. ${ }^{44,45}$ Overproduction of Th2 cytokines is also a phenotype of $\mathrm{CD}^{+} \mathrm{T}$ cells isolated from Mapk8 ${ }^{-/-}$mice and transgenic mice engineered to overproduce JunB. ${ }^{46,47}$ Map3k $1^{\triangle K D}$, Mapk $^{-1-}$ and Itchy Th2 cells all produce similarly deficient responses in a mouse T-cell allergy model. ${ }^{48}$ As well as having an important role in $\mathrm{CD}^{+}{ }^{+} \mathrm{T}$-cell differentiation the Map3k1 kinase domain also has a negative regulatory role in the proliferative-expansion of $\mathrm{CD}^{+} \mathrm{T}^{\mathrm{C}}$ cells. ${ }^{49}$

Either Map3k1 or Mapk8-Map2k7 fusion protein can enhance Itch E3 Ub ligase activity toward its substrate JunB in HEK 293 cells. $^{43,45}$ After T-cell receptor engagement Map3k1-dependent Mapk8 signaling is activated in T cells and Itch undergoes Mapk8-mediated phosphorylation of Ser199, Ser232 and Thr222 within the Itch Pro-rich region. ${ }^{50}$ Direct Itch phosphorylation by Mapk8 disrupts an inhibitory interaction that occurs between the Itch WW domains, that mediate protein-protein interactions, and the HECT domain, the E3 Ub ligase, and this change in Itch conformation leads to the significantly increased activity of the HECT domain. ${ }^{50}$ Map3k1 and Itch also form a complex within T cells as Itch is activated by Mapk8 phosphorylation. ${ }^{51}$

Despite Map3k1 $1^{\triangle K D}$ T cells displaying skewed Th2 cytokine production, Map $3 k 1^{\triangle K D}$ mice show both significantly reduced germinal center formation within their secondary lymphoid tissues and production of antibodies in response to thymusdependent, but not thymus-independent, antigens. ${ }^{52-54}$ As suggested by early work measuring Map3k1 activation in B-cell lines following CD40 engagement with antibodies, Map3k1 was found to be necessary for CD40 ligand (CD40L, also known as CD154) -mediated activation of Mapk8/9 and Mapk14 in B cells. ${ }^{15,52,54}$ Map3k $1^{\Delta K D} \mathrm{~B}$ cells have significantly reduced C-Jun phosphorylation and defective expression of both Cyclin D2, a regulator of cyclindependent kinases, and Activation-induced deaminase, a protein important in antibody diversity, that likely explains the poor humoral immune responses seen in Map3k $1^{\triangle K D}$ mice. ${ }^{52,54}$

Following the engagement of CD40 by CD40L, and also many other Tnfrs by their ligands, Tnf receptor-associated factor (Traf) 2 (Traf2), Traf3, Ub-conjugating enzyme E2 N (Ube2N, also known as Ubc13), cellular inhibitor of apoptosis proteins 1 and 2 (c-lap1/2), Ikk $\gamma$ and Map3k1 are recruited rapidly to the CD40 receptor. ${ }^{52,53}$ Traf2, Ube2N and Ikky are all necessary components for both the assembly of the signal transduction complex at the receptor and the activation of Map3k1 and its downstream Mapks. ${ }^{52,53}$ The CD40 Mapk signaling complex is inactive at the receptor, but the complex then translocates from the CD40 receptor into the cytosol to become active after the transfer of Lys48-linked poly-Ub by c-lap1/2 onto Traf3 and the subsequent degradation of Traf3 by the proteasome. ${ }^{52,53}$

\section{Role in Stem Cells and Cardiac Myocytes}

Initial analyses using Map3 $k 1^{-1}$ ES cells showed they are deficient in Mapk activation in response to a wide variety of stimuli, including microtubule disrupting drugs, low temperature shock, hyperosmotic stress and growth factors present within the serum. ${ }^{29,30}$ As stated above, Map3k1 $1^{-/}$ES cells have a greater propensity to enter apoptosis following hyperosmotic stress or treatment with microtubule disrupting drugs than wild-type (WT) ES cells. ${ }^{29,30}$ Map3k $1^{\Delta K D}$ ES cells display reduced Mapk8/9 activation in response to pro-

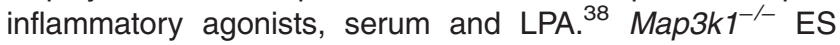
cell-derived cardiac myocytes display increased sensitivity to apoptosis following hydrogen peroxide-induced stress. In response to hydrogen peroxide-induced stress $M a p 3 k 1^{-1-}$ ES cell-derived cardiac myocytes show reduced Mapk8/9 phosphorylation. $^{31}$

Map3k1 can be activated by heart-restricted expression of $\mathrm{Gaq}$, a heterotrimeric $\mathrm{G}$ protein subunit, in transgenic mice. ${ }^{55}$ Map3 $\mathrm{k}^{-/}$ES cell-derived cardiac myocytes have reduced Mapk8/9 phosphorylation following treatment with the $a_{1}$ Adrenergic receptor agonist phenylephrine. ${ }^{55}$ Map3 $k 1^{-1-}$ mice display increased cardiac mass, larger cardiac myocytes, elevated Atrial natriuretic factor (Anf) expression, impaired Mapk8/9 phosphorylation by Gaq and improved ventricular function. ${ }^{55}$ Map3 $k 1^{-1-}$ mice also show reduced Mapk8/9 phosphorylation following transverse aortic constriction, an indicator that Map3k1 may regulate Mapk8/9 activation following cardiac pressure overload, where cardiac muscle is forced to contract while undergoing excessive afterload. ${ }^{56}$ Pressure overload causes significant cardiac hypertrophy and increased expression of Anf in Map3k $1^{-1-}$ mice, which also show higher mortality and a greater ratio of lung to body mass. ${ }^{56}$ Map3k1 may be required for pressure overload-induced Mapk8/9 activation and enhanced production of Tnf- $\alpha$ and Tgf- $\beta$ cytokines. ${ }^{56}$ Map3k1 can function in the cardiovascular system to promote cardiac myocyte cell survival, reduce inflammation and protect against cardiac failure. $^{56}$

\section{Roles in Cancer}

Early analyses identified a potential role for MAP3K1 in Androgen receptor signaling in prostate cancer cell lines. ${ }^{57}$ The Androgen receptor-positive LNCaP cell line, derived from Androgen-sensitive human prostate adenocarcinoma cells, undergoes apoptosis when transduced with a retroviral vector that overexpresses the Map3k1 kinase domain. ${ }^{57}$ By contrast, Androgen receptor-negative DU145 cells and PC3 cells do not enter apoptosis when transduced with retrovirus expressing the Map3k1 kinase domain. ${ }^{57}$ Co-transduction of the Androgen receptor and Map3k1 kinase domain into DU145 cells leads to apoptosis. ${ }^{57}$ Conversely, transfection of kinaseinactive Map3k1 expressing cDNA into BxPC-3, PANC-1, MIAPaCa-2 and AsPC-1 pancreatic cancer cell lines promoted cell death in pancreatic cancer cell lines, suggesting a role for Map3k1 kinase domain signaling in tumor cell survival. ${ }^{58}$

Small interfering RNA knockdown of MAP3K1 expression in the invasive human adenocarcinoma cell line MDA-MB-231, that contains an aberrant $W n t 7 b$ oncogene, causes a significant reduction in urokinase-type plasminogen activator (UPA), a serine protease whose expression can correlate with tumor malignancy, activity. ${ }^{59,60}$ Knockdown of MAP3K1 in MDA-MB-231 cells causes reduced migration towards serum growth factors in transwell migration assays when compared with MDA-MB-231 cells transfected with a control small 
interfering RNA. ${ }^{60}$ These results suggest a potential role for MAP3K1 in regulating both tumor malignancy and cancer cell invasion into normal tissues.

Map3 $k 1^{-/}$mice crossed to a polyoma virus middle $\mathrm{T}$ antigen (PyMT) transgene under control of the mouse mammary tumor virus long terminal repeat (MMTV LTR) show significantly delayed development of lung metastases. ${ }^{60}$ The dissemination of cancerous cells from Map $3 \mathrm{k} 1^{-/}$mammary tumors is reduced, though the eventual formation of lung metastases still occurs in mice. ${ }^{60}$ The delay in the development of lung metastasis observed in Map3k $1^{-/-}$mice is perhaps caused by the reduced integrity of the basement membranes surrounding the Map3k $1^{-1-}$ mammary tumors. ${ }^{60}$ Map3k1 may regulate both proteolytic degradation and migration of tumor cells in mice. ${ }^{60}$

Screening for breast cancer susceptibility alleles has identified, amongst several genes, MAP3K1 as a causative gene for breast cancer. ${ }^{61}$ In fact, $\sim 12 \%$ of all luminal A breast cancer tumors contain mutations within MAP3K1 or MAP2K4, kinases that can regulate MAPK8/9 and MAPK14 signaling. ${ }^{62}$ Almost all breast cancer MAP $3 K 1$ and MAP2K 4 mutations are found within luminal A tumors, and MAP3K1 and MAP2K4 mutations are largely mutually exclusive of each other. ${ }^{62}$ Although the role for MAP3K1 in some forms of breast cancer is substantiated by numerous reports, ${ }^{61,63-68}$ the importance of MAP3K1 in other cancers is far less certain. A recent lowcopy transposon mutagenesis screening methodology in mice identified Map3k1 as a potentiator of melanoma. ${ }^{69}$ Point mutations within introns 9 and 10 of Map3k1 produce truncated forms of Map3k1 that lack the N-terminal regulatory region. ${ }^{69} \mathrm{~N}$-terminal truncation of Map3k1 leads to enhanced Mapk $1 / 3$ phosphorylation in melanoma tumors, and provides a possible mechanism to explain how Map3k1 may drive malignancy in melanoma. ${ }^{69}$ It is also notable that host immunodeficiency may also contribute to the prognosis of Map3k1-dependent cancers. ${ }^{70}$

\section{New Insights into Map3k1 PHD Motif Signaling by Gene Knockin of Map3k1}

To better understand the functions of the PHD motif in mammalian biology we first modeled the Map3k1 PHD upon the known structure of the Deltex 2 Really Interesting New Gene (RING), which has homology with the Map3k1 PHD motif, and mutated conserved residues within the E2 binding region of the PHD structure (Cys438Ala and Ile440Ala, the Map3k1 mPHD mutation) to inactivate the E3 Ub ligase, and the Map3k1 mPHD mutation does not significantly reduce kinase domain activity. ${ }^{34,71,72}$ Transfection of Map3k1 mPHD expressing cDNA into HEK 293 cells revealed that the Map3k1 mPHD protein displays significantly impaired Map3k1 autoubiquitination. ${ }^{19,33,72}$ Analysis of the E2 conjugating enzymes that can act in concert with Ube1 and the Map3k1 PHD revealed that Ub-conjugating enzyme E2D (Ube2D) 2 (Ube2D2), Ube2D3 and Ube2N:Ub-conjugating enzyme E2 variant 1 (Ube2V1) can all mediate Map3k1 autoubiquitination. ${ }^{72}$ Previously it was known that the Map3k1 PHD may utilize Ube2D2 to transfer poly-Ub onto Mapk1. ${ }^{19,33}$ Immunoblotting with anti-Lys63-linked Ub monoclonal antibodies demonstrated that the Map3k1 PHD motif largely forms
Lys63-linked poly-Ub chains as opposed to linear poly-Ub chains. ${ }^{72}$ Lys63-linked poly-Ub is efficiently removed from Map3k1 by the deubiquitinating enzymes Ub-specific protease 2,7 and $8 .^{72}$

Mutation of Map3k1 alleles to express the Map3k1 mPHD (encoded by Map3k $1^{m P H D}$ ) revealed that Map3k $1^{m P H D}$ ES cells do not exhibit defective Mapk1 expression following longterm hyperosmotic stress as has previously been suggested by the overexpression analysis in cell lines. ${ }^{19,33,72}$ This finding suggested that while Mapk1 is a substrate for Map3k1 PHD ubiquitination in cells, Map3k1 may not have a critical role in regulating Mapk1 by poly-Ub and there may also be other Mapk1 E3 Ub ligases that perform this function in cells undergoing hyperosmotic stress. ${ }^{19,33}$ However, the kinetics of the full-length Map3k1 degradation in response to hyperosmotic stress are altered in Map3k $1^{m P H D}$ ES cells. ${ }^{72}$ Our findings suggested that the Map3k1 PHD can critically regulate the rapidity of its own degradation while undergoing hyperosmotic stress by auto-ubiquitination, but that other E3 Ub ligases (e.g. Deltex family E3 Ub ligases) may also act as E3 Ub ligases toward Map3k1 following hyperosmotic stress in the absence of a functional PHD motif. ${ }^{72,73}$ Surprisingly, we identified a defective Mapk activation in Map3k $1^{m P H D}$ ES cells following their treatment with microtubule disrupting drugs and the cytokines Tgf- $\beta$ or Egf, suggesting that the Map3k1 PHD, in fact, has an unexpected and critical role in regulating Mapk activation. ${ }^{72}$

Neither of the known Map3k1 PHD substrates (Mapk1 and c-Jun) provide an obvious explanation for the new critical role found for the PHD motif in cytokine-induced Mapk activation, ${ }^{19,32,33,61}$ so we searched for novel substrates using a high throughput screening approach that analyzed a library of over 9400 human full-length proteins. ${ }^{74,75}$ Our Ub protein array screening methodology identified 82 new proteins as potential substrates for a ubiquitination reaction containing Ube1, Ube2N:Ube2V1 and the Map3k1 PHD motif. ${ }^{72}$ Many of the PHD motif substrates are molecular scaffold proteins involved in signal transduction, and bioinformatics analysis suggested that Tgf $\beta$ activated kinase 1-binding protein (Tab) 1 (Tab1) was critical for Tgf- $\beta$-induced Mapk activation. $^{72,76}$

Ubiquitination assays by orthogonal approaches confirmed that the Map3k1 PHD can transfer Lys63-linked poly-Ub onto recombinant Tab1, and also other scaffold proteins, namely Traf2, TNFAIP3 interacting protein (Tnip) 1 (Tnip1), Tnip2 and Signal transducing adapter molecule 1 (Stam1). ${ }^{72}$ Of these Map3k1 PHD motif substrates, and after immunoprecipitation from the WT and Map3k $1^{m P H D}$ ES cells stimulated by Tgf- $\beta$, only Tab1 was found to be significantly ubiquitinated by Lys63linked poly-Ub in WT and not Map3k $1^{m P H D}$ ES cells. ${ }^{72}$ Generation of $\mathrm{Tab}^{-/-}$ES cells revealed that they, like Map3k $1^{m P H D}$ ES cells, are deficient in Egf- and Tgf- $\beta$-induced Mapk and Map3k7 (also known as Tak1) activation. ${ }^{72}$ Mapping of the Tab1 ubiquitination sites mediated by the Map3k1 PHD and Ube2N:Ube2V1 identified Lys294, Lys319, Lys335 and Lys350 as being important for Tab1 ubiquitination by the PHD motif. Map3k1 can interact with and transfer Lys63-linked poly-Ub onto Tab1 by its PHD motif to potentiate the proteinprotein interaction between Tab1 and Map3k7. ${ }^{72}$ Tab2, though not itself a Map3k1 PHD substrate, can be recruited into the 
Tab1:Map3k1 Ub complex to form a ternary complex that is dependent upon the Tab2 zinc finger $(\mathrm{ZnF})$, a motif that can interact with proteins that possess Lys63-linked poly-Ub chains. $^{72,77}$ Recruitment of Tab2 into the Map3k1:Tab1 signaling complex may facilitate a further downstream signaling from Tgf- $\beta$ receptors (Tgf $\beta$ rs) and Egf receptors (Egfrs) (Figure 2). The formation of a Ub signaling complex between Tab1:Map3k1:Map3k7 offers a plausible explanation for why Map3k $1^{m P H D}$ ES cells or WT ES cells treated with the Map3k7 chemical inhibitor (5Z)-7-oxozeaenol both lose Mapk activation following stimulation by Tgf- $\beta$ or Egf cytokines. ${ }^{72}$ Chemical inhibition using the Ube2N inhibitor NSC697923 demonstrates that Ube2N is also critical for Map3k7 and Mapk activation in ES cells following treatment with Tgf- $\beta$ or Egf, and Ube2N is also important for Tgf- $\beta$-induced Mapk activation in breast cancer cells. ${ }^{72,78}$ However, unlike CD40 signaling in B cells, where Traf2 is critical for Mapk8/9 and Mapk14 activation, ${ }^{52,53}$ instead for Tgf $\beta$ rs Traf6 is critical for Mapk activation. ${ }^{79}$ The identification of a new PHD motif substrate that forms the lynchpin between Tgf- $\beta$-dependent Mapk pathway activation and stem cell differentiation further complicates the role for the Map3k1 PHD motif in apoptosis, and suggests that under conditions of cell death induced by hyperosmotic stress Map3k1 may silence Mapk signaling, while when stimulated by cytokines the Map3k1 PHD plays a role in promoting cell survival and differentiation by activating Mapks (Figure 3).

Both Tab1 and the Map3k1 PHD can negatively regulate neuroectoderm genes and enhance long-term expression of mesoderm genes in ES cells as they differentiate from pluripotent stem cells into embryoid bodies in cell culture. ${ }^{72}$ Mapk8 ${ }^{-/} / \mathrm{Mapk}^{-/}$double deficiency in ES cells has demonstrated that Mapk8/9 are important for driving ES cell differentiation. ${ }^{80}$ Mapk14 is also known to have an important role in ES cell differentiation by regulating neuroectoderm and mesoderm formation. ${ }^{81,82}$ Despite these results no compound mutant Mapk8 ${ }^{-/} /$Mapk9 $^{-/-}$- and Mapk $14^{-/-}$-deficient ES cells have been generated and analyzed to date that would produce the similar differentiation defects observed in Map3k $1^{m P H D}$ or $\mathrm{Tab1}^{-1-}$ ES cells. Transplantation of immunodeficient host mice with either $T a b 1^{-1-}$ or Map3k $1^{\mathrm{mPHD}}$ ES cells causes aberrant tumors to form, and these have altered tissue composition and are of smaller mass and size. ${ }^{72}$ Add-back of Tab1, but not lysine-mutated (Lys294Ala, Lys319Ala, Lys335Ala and Lys350Ala) Tab1, into $\mathrm{Tab1}^{-1-}$ ES cells restores normal ES cell differentiation and tumorigenesis, demonstrating that the lysines in Tab1 ubiquitinated by the Map3k1 PHD motif are critically important for ES cell differentiation and tumor formation in mice. ${ }^{72}$

Analysis of Map3k $1^{m P H D}$ knockin mice is complicated by their early lethality during embryogenesis, a more severe phenotype than the partial lethality observed in Map3k $1^{\Delta K D}$ mice $^{72,83}$ Indeed, the combination of aberrant regulation of the Ub-proteasome system and defects in Mapk signaling provide a plausible explanation for the more severe phenotypes of Map3k1 $1^{m P H D}$ mice. $^{19,33}$ However, mature Map3k $1^{\mathrm{mPHD} /+}$ mice are viable for phenotypic analysis and have demonstrated that signal transduction by the Map3k1 PHD motif is critical for B-cell development beyond the Pro-Bcell stage, T-cell receptor signal transduction and Itch phosphorylation within the pro-rich region , protecting cardiac tissue and maintaining the Leydig cell population within testis. $^{72}$

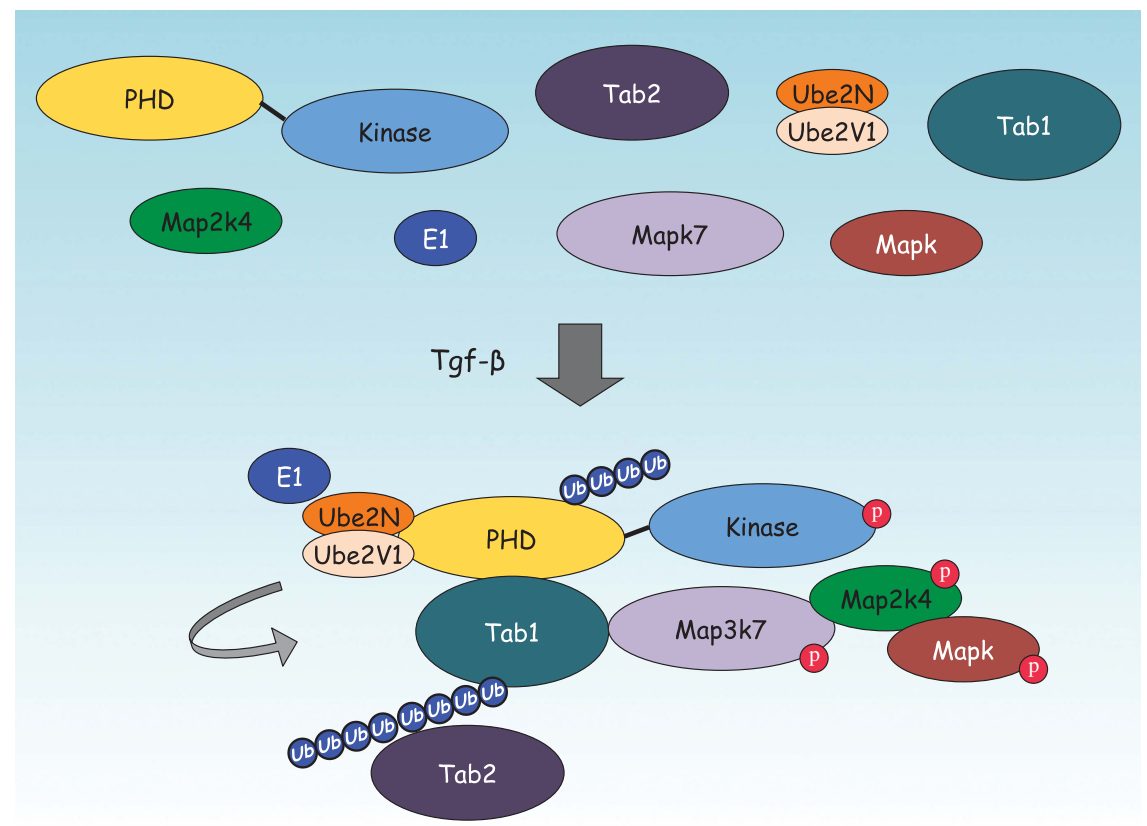

Figure 2 The Map3k1 PHD motif regulates Tabs in response to cytokine stimulation. Following Tgf- $\beta$ treatment, the Map3k1 PHD motif binds and transfers Lys63-linked polyUb onto Tab1 to enhance Map3k7 activation. Tab2, although not a Map3k1 PHD motif substrate, can be recruited to the Map3k1:Map3k7 Ub signaling complex by the Ub binding $\mathrm{ZnF}$ motif of Tab2. 


\section{Summary}

From its initial discovery as the second Map2k1 kinase to defining the role of Map3k1 signal transduction in tumorigenesis and breast cancer, the road to understanding the intricate mechanisms of Map3k1 signaling has seemed to go on forever (Figure 4). Our new insights into the role of the Map3k1 PHD motif provide a fresh perspective into how Map3k1 signaling can regulate both the Ub-proteasome and protein phosphorylation systems. Analysis of Map3k1 domain-specific signaling has now revealed several important brand-new insights. At cytokine receptors and in response to microtubule disruption the Map3k1 PHD motif and kinase domain are both required

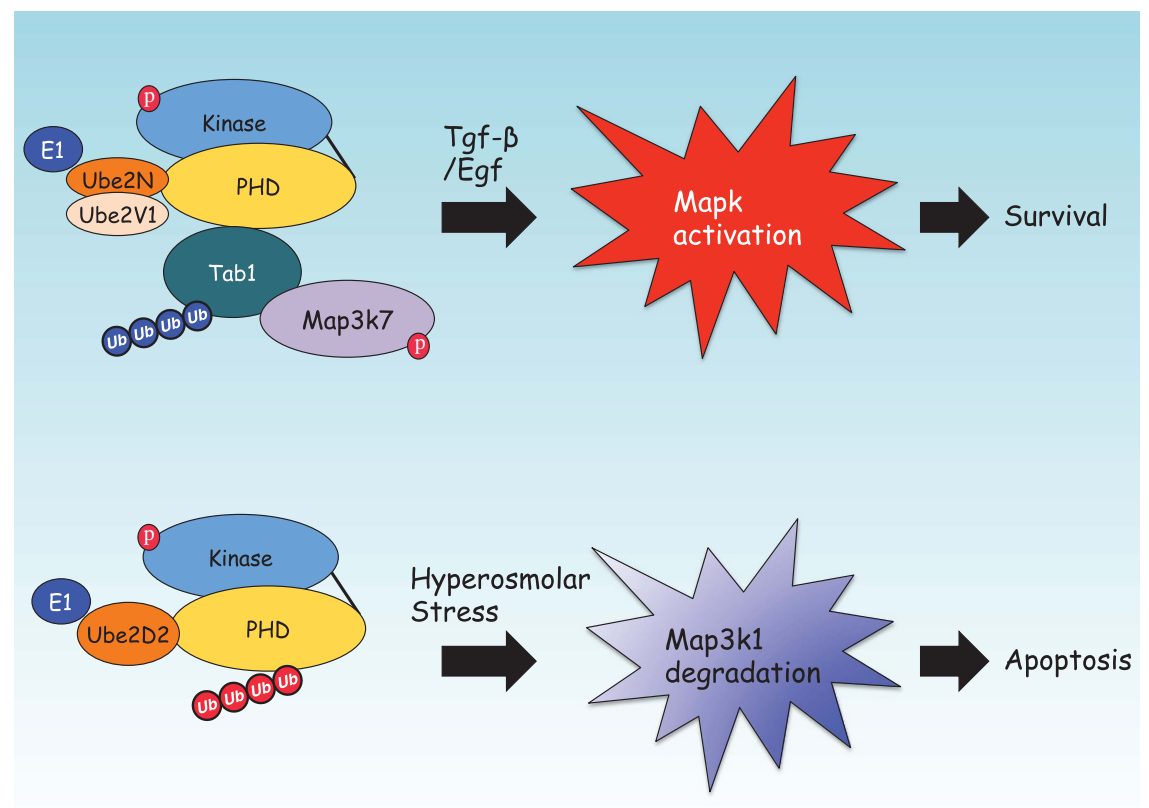

Figure 3 The signal transduction role for the Map3k1 PHD motif during cell survival or apoptosis. In response to Tgf- $\beta$ or Egf cytokines Map3k1 ubiquitinates Tab1 with Lys63linked poly-Ub to activate Map3k7 and Mapk. This promotes both the differentiation and survival of stem cells. In response to hyperosmotic stress, stem cells enter apoptosis and the Map3k1 PHD motif transfers Lys48-linked poly-Ub onto itself, in conjunction with other E3 Ub ligases, to potentiate the degradation of the Mapk signaling cascade by the proteasome.

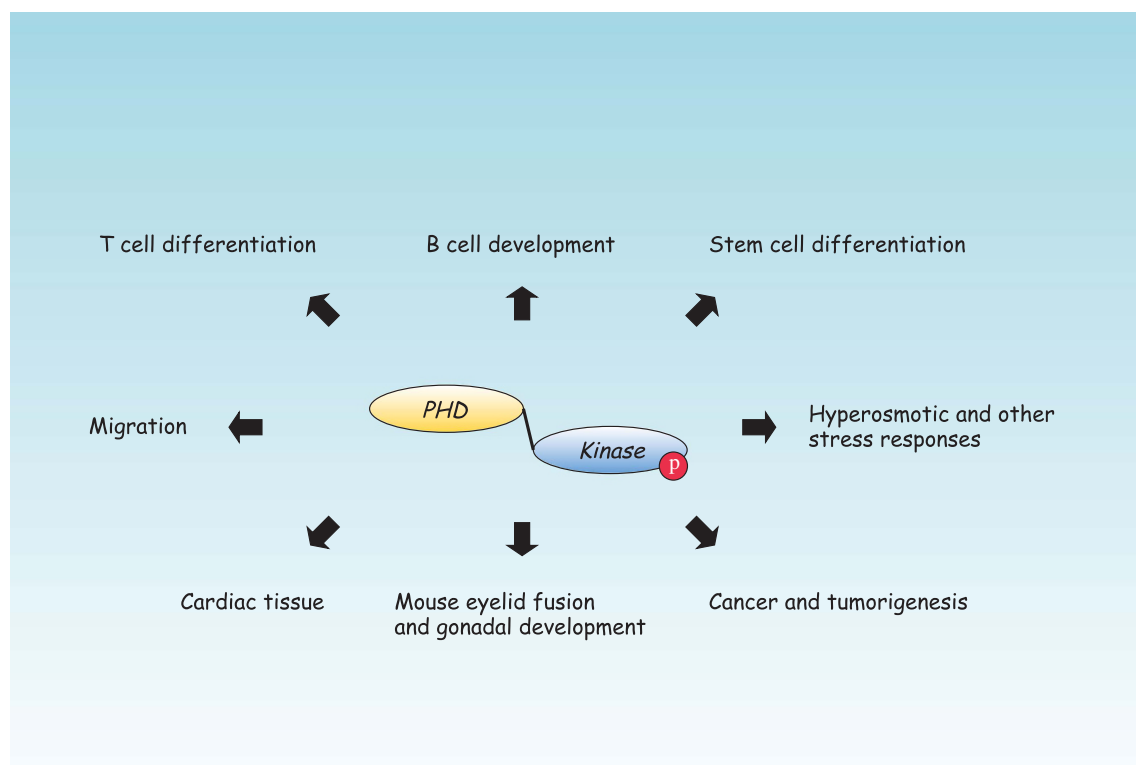

Figure 4 Tissues, cells and diseases where mouse or human genetics have demonstrated Map3k1 signaling to be of importance.,30,45,52,99 
for Mapk activation. But, while the Map3k1 kinase domain is required for hyperosmotic stress-dependent Mapk activation, the PHD motif is dispensable for Mapk activation under this circumstance, and instead enhances the kinetics of full-length Map3k1 degradation. Analysis of the Map3k1 PHD by mouse genetics has also demonstrated that, like the Map3k1 kinase domain, the PHD motif is important for lymphocyte T-cell receptor signaling, cardiac tissue damage and stem cells. However, it is notable that disruption of the Map3k1 PHD motif has a more dramatic effect upon B-cell development than kinase domain ablation, the degree of embryonic lethality encountered is more severe in Map3k $1^{m P H D}$ than Map3k $1^{\triangle K D}$ mice and there are more severe mesoderm and neuroectoderm differentiation defects in Map3k $1^{m P H D}$ than Map3k $1^{\triangle K D}$ stem cells. As such, modulation of the Map3k1 PHD motif may eventually provide an attractive alternative target to the kinase domain for future drug discovery. ${ }^{84}$

\section{Conflict of Interest}

The authors declare no conflict of interest.

Acknowledgements. Our research was supported by grants from the Wellcome Trust (WT090939MA) and Cancer Research UK (C26616/A12679).

1. Raman M, Chen W, Cobb MH. Differential regulation and properties of MAPKs. Oncogene 2007; 26: 3100-3112.

2. Xia Y, Karin M. The control of cell motility and epithelial morphogenesis by Jun kinases. Trends in cell biology 2004; 14: 94-101.

3. Karin M, Gallagher E, From JNK. to pay dirt: jun kinases, their biochemistry, physiology and clinical importance. IUBMB Life 2005; 57: 283-295.

4. Weston CR, Davis RJ. The JNK signal transduction pathway. Curr Opin Cell Biol 2007; 19 : 142-149.

5. Lange-Carter CA, Pleiman CM, Gardner AM, Blumer KJ, Johnson GL. A divergence in the MAP kinase regulatory network defined by MEK kinase and Raf. Science 1993; 260 315-319.

6. Blumer KJ, Johnson GL, Lange-Carter CA. Mammalian mitogen-activated protein kinase kinase kinase (MEKK) can function in a yeast mitogen-activated protein kinase pathway downstream of protein kinase C. Proc Natl Acad Sci USA 1994; 91: 4925-4929.

7. Gardner AM, Vaillancourt RR, Lange-Carter CA, Johnson GL. MEK-1 phosphorylation by MEK kinase, Raf, and mitogen-activated protein kinase: analysis of phosphopeptides and regulation of activity. Mol Biol Cell 1994; 5: 193-201.

8. Yan M, Templeton DJ. Identification of 2 serine residues of MEK- 1 that are differentially phosphorylated during activation by raf and MEK kinase. J Biol Chem 1994; 269: 19067-19073.

9. Gallagher ED, Xu S, Moomaw C, Slaughter CA, Cobb MH. Binding of JNK/SAPK to MEKK1 is regulated by phosphorylation. J Biol Chem 2002; 277: 45785-45792.

10. Minden A, Lin A, McMahon M, Lange-Carter C, Derijard B, Davis RJ et al. Differential activation of ERK and JNK mitogen-activated protein kinases by Raf-1 and MEKK. Science 1994; 266: 1719-1723

11. Yan M, Dai T, Deak JC, Kyriakis JM, Zon LI, Woodgett JR et al. Activation of stress-activated protein kinase by MEKK1 phosphorylation of its activator SEK1. Nature 1994; 372: 798-800.

12. Xia Y, Wu Z, Su B, Murray B, Karin M. JNKK1 organizes a MAP kinase module through specific and sequential interactions with upstream and downstream components mediated by its amino-terminal extension. Genes Dev 1998; 12: 3369-3381.

13. Lin A, Minden A, Martinetto H, Claret FX, Lange-Carter C, Mercurio F et al. Identification of a dual specificity kinase that activates the Jun kinases and p38-Mpk2. Science 1995; 268: 286-290.

14. Winston BW, Lange-Carter CA, Gardner AM, Johnson GL, Riches DW. Tumor necrosis factor alpha rapidly activates the mitogen-activated protein kinase (MAPK) cascade in a MAPK kinase kinase-dependent, c-Raf-1-independent fashion in mouse macrophages. Proc Natl Acad Sci USA 1995; 92: 1614-1618.

15. Sakata N, Patel HR, Terada N, Aruffo A, Johnson GL, Gelfand EW. Selective activation of c-Jun kinase mitogen-activated protein kinase by CD40 on human B cells. J Biol Chem 1995; 270: 30823-30828

16. Xu S, Robbins DJ, Christerson LB, English JM, Vanderbilt CA, Cobb MH. Cloning of rat MEK kinase $1 \mathrm{CDNA}$ reveals an endogenous membrane-associated 195-kDa protein with a large regulatory domain. Proc Natl Acad Sci USA 1996; 93: 5291-5295.

17. Hirano M, Osada S, Aoki T, Hirai S, Hosaka M, Inoue J et al. MEK kinase is involved in tumor necrosis factor alpha-induced NF-kappaB activation and degradation of IkappaB-alpha. J Biol Chem 1996; 271: 13234-13238.
18. Lee FS, Hagler J, Chen ZJ, Maniatis T. Activation of the IkappaB alpha kinase complex by MEKK1, a kinase of the JNK pathway. Cell 1997; 88: 213-222.

19. Lu Z, Xu S, Joazeiro C, Cobb MH, Hunter T. The PHD domain of MEKK1 acts as an E3 ubiquitin ligase and mediates ubiquitination and degradation of ERK1/2. Mol Cell 2002; 9 : 945-956.

20. Rieger MA, Duellman T, Hooper C, Ameka M, Bakowska JC, Cuevas BD. The MEKK1 SWIM domain is a novel substrate receptor for c-Jun ubiquitylation. Biochem J 2012; 445: 431-439.

21. Johnson NL, Gardner AM, Diener KM, Lange-Carter CA, Gleavy J, Jarpe MB et al. Signal transduction pathways regulated by mitogen-activated/extracellular response kinase kinase kinase induce cell death. J Biol Chem 1996; 271: 3229-3237.

22. Widmann C, Johnson NL, Gardner AM, Smith RJ, Johnson GL. Potentiation of apoptosis by low dose stress stimuli in cells expressing activated MEK kinase 1. Oncogene 1997; 15: 2439-2447.

23. Widmann C, Gerwins P, Johnson NL, Jarpe MB, Johnson GL. MEK kinase 1, a substrate for DEVD-directed caspases, is involved in genotoxin-induced apoptosis. Mol Cell Biol 1998; 18: 2416-2429.

24. Deak JC, Cross JV, Lewis M, Qian Y, Parrott LA, Distelhorst CW et al. Fas-induced proteolytic activation and intracellular redistribution of the stress-signaling kinase MEKK1. Proc Natl Acad Sci USA 1998; 95: 5595-5600.

25. Cardone MH, Salvesen GS, Widmann C, Johnson G, Frisch SM. The regulation of anoikis: MEKK-1 activation requires cleavage by caspases. Cell 1997; 90: 315-323.

26. Gibson S, Widmann C, Johnson GL. Differential involvement of MEK kinase 1 (MEKK1) in the induction of apoptosis in response to microtubule-targeted drugs versus DNA damaging agents. J Biol Chem 1999; 274: 10916-10922.

27. Chang L, Kamata H, Solinas G, Luo JL, Maeda S, Venuprasad K et al. The E3 ubiquitin ligase itch couples JNK activation to TNFalpha-induced cell death by inducing c-FLIP(L) turnover. Cell 2006; 124: 601-613.

28. Fuchs SY, Adler V, Pincus MR, Ronai Z. MEKK1/JNK signaling stabilizes and activates p53. Proc Natl Acad Sci USA 1998; 95: 10541-10546.

29. Yujiri T, Fanger GR, Garrington TP, Schlesinger TK, Gibson S, Johnson GL. MEK kinase 1 (MEKK1) transduces c-Jun NH2-terminal kinase activation in response to changes in the microtubule cytoskeleton. J Biol Chem 1999; 274: 12605-12610.

30. Yujiri T, Sather S, Fanger GR, Johnson GL. Role of MEKK1 in cell survival and activation of JNK and ERK pathways defined by targeted gene disruption. Science 1998; 282 : 1911-1914.

31. Minamino T, Yujiri T, Papst PJ, Chan ED, Johnson GL, Terada N. MEKK1 suppresses oxidative stress-induced apoptosis of embryonic stem cell-derived cardiac myocytes. Proc Natl Acad Sci USA 1999; 96: 15127-15132.

32. Xia Y, Wang J, Xu S, Johnson GL, Hunter T, Lu Z. MEKK1 mediates the ubiquitination and degradation of C-Jun in response to osmotic stress. Mol Cell Biol 2007; 27: 510-517.

33. Lu Z, Hunter T. Degradation of activated protein kinases by ubiquitination. Annu Rev Biochem 2009; 78: 435-475.

34. Dikic I, Robertson M. Ubiquitin ligases and beyond. BMC biology 2012; 10: 22

35. Xia Y, Yang W, Bu W, Ji H, Zhao X, Zheng Y et al. Differential regulation of c-Jun protein plays an instrumental role in chemoresistance of cancer cells. J Biol Chem 2013; 288 : 19321-19329.

36. Tricker E, Arvand A, Kwan R, Chen GY, Gallagher E, Cheng G. Apoptosis induced by cytoskeletal disruption requires distinct domains of MEKK1. PIOS One 2011; 6: e17310.

37. Steed E, Elbediwy A, Vacca B, Dupasquier S, Hemkemeyer SA, Suddason T et al. MarvelD3 couples tight junctions to the MEKK1-JNK pathway to regulate cell behavior and survival. J Cell Biol 2014; 204: 821-838.

38. Xia Y, Makris C, Su B, Li E, Yang J, Nemerow GR et al. MEK kinase 1 is critically required for C-Jun $\mathrm{N}$-terminal kinase activation by proinflammatory stimuli and growth factor-induced cell migration. Proc Natl Acad Sci USA 2000; 97: 5243-5248.

39. Zhang L, Wang W, Hayashi Y, Jester JV, Birk DE, Gao M et al. A role for MEK kinase 1 in TGF-beta/activin-induced epithelium movement and embryonic eyelid closure. EMBO J 2003; 22: 4443-4454

40. Cuevas BD, Abell AN, Witowsky JA, Yujiri T, Johnson NL, Kesavan K et al. MEKK1 regulates calpain-dependent proteolysis of focal adhesion proteins for rear-end detachment of migrating fibroblasts. EMBO J 2003; 22: 3346-3355.

41. Christerson LB, Vanderbilt CA, Cobb MH. MEKK1 interacts with alpha-actinin and localizes to stress fibers and focal adhesions. Cell Motil Cytoskeleton 1999; 43 : 186-198.

42. Glading A, Bodnar RJ, Reynolds IJ, Shiraha H, Satish L, Potter DA et al. Epidermal growth factor activates m-calpain (calpain II), at least in part, by extracellular signal-regulated kinase-mediated phosphorylation. Mol Cell Biol 2004; 24: 2499-2512.

43. Gao M, Labuda T, Xia Y, Gallagher E, Fang D, Liu YC et al. Jun turnover is controlled through JNK-dependent phosphorylation of the E3 ligase Itch. Science 2004; 306: 271-275.

44. Fang D, Elly C, Gao B, Fang N, Altman Y, Joazeiro C et al. Dysregulation of T lymphocyte function in itchy mice: a role for Itch in TH2 differentiation. Nature Immunol 2002; 3 : 281-287.

45. Gao M, Karin M. Regulating the regulators: control of protein ubiquitination and ubiquitin-like modifications by extracellular stimuli. Mol Cell 2005; 19: 581-593.

46. Dong C, Yang DD, Tournier C, Whitmarsh AJ, Xu J, Davis RJ et al. JNK is required for effector T-cell function but not for T-cell activation. Nature 2000; 405: 91-94.

47. Li B, Tournier C, Davis RJ, Flavell RA. Regulation of IL-4 expression by the transcription factor JunB during T helper cell differentiation. EMBO J 1999; 18: 420-432. 
48. Venuprasad K, Elly C, Gao M, Salek-Ardakani S, Harada Y, Luo JL et al. Convergence of Itch-induced ubiquitination with MEKK1-JNK signaling in Th2 tolerance and airway inflammation. J Clin Invest 2006; 116: 1117-1126.

49. Labuda T, Christensen JP, Rasmussen S, Bonnesen B, Karin M, Thomsen AR et al. MEK kinase 1 is a negative regulator of virus-specific CD8(+) T cells. Eur J Immunol 2006; 36 : 2076-2084.

50. Gallagher E, Gao M, Liu YC, Karin M. Activation of the E3 ubiquitin ligase Itch through a phosphorylation-induced conformational change. Proc Natl Acad Sci USA 2006; 103: 1717-1722.

51. Enzler T, Chang X, Facchinetti V, Melino G, Karin M, Su B et al. MEKK1 binds HECT E3 ligase Itch by its amino-terminal RING motif to regulate Th2 cytokine gene expression. J Immunol 2009; 183: 3831-3838.

52. Karin M, Gallagher E. TNFR signaling: ubiquitin-conjugated TRAFfic signals control stopand-go for MAPK signaling complexes. Immunol Rev 2009; 228: 225-240.

53. Matsuzawa A, Tseng PH, Vallabhapurapu S, Luo JL, Zhang W, Wang $\mathrm{H}$ et al. Essential cytoplasmic translocation of a cytokine receptor-assembled signaling complex. Science 2008; 321: 663-668.

54. Gallagher E, Enzler T, Matsuzawa A, Anzelon-Mills A, Otero D, Holzer R et al. Kinase MEKK1 is required for CD40-dependent activation of the kinases Jnk and p38, germinal center formation, B cell proliferation and antibody production. Nature Immunol 2007; 8: $57-63$.

55. Minamino T, Yujiri T, Terada N, Taffet GE, Michael LH, Johnson GL et al. MEKK1 is essential for cardiac hypertrophy and dysfunction induced by Gq. Proc Natl Acad Sci USA 2002; 99: 3866-3871.

56. Sadoshima J, Montagne O, Wang Q, Yang G, Warden J, Liu J et al. The MEKK1-JNK pathway plays a protective role in pressure overload but does not mediate cardiac hypertrophy. J Clin Invest 2002; 110: 271-279.

57. Abreu-Martin MT, Chari A, Palladino AA, Craft NA, Sawyers CL. Mitogen-activated protein kinase kinase kinase 1 activates androgen receptor-dependent transcription and apoptosis in prostate cancer. Mol Cell Biol 1999; 19: 5143-5154.

58. Hirano T, Shino Y, Saito T, Komoda F, Okutomi Y, Takeda A et al. Dominant negative MEKK1 inhibits survival of pancreatic cancer cells. Oncogene 2002; 21: 5923-5928.

59. Blasi F, Vassalli JD, Dano K. Urokinase-type plasminogen activator: proenzyme, receptor, and inhibitors. J Cell Biol 1987; 104: 801-804.

60. Cuevas BD, Winter-Vann AM, Johnson NL, Johnson GL. MEKK1 controls matrix degradation and tumor cell dissemination during metastasis of polyoma middle-T driven mammary cancer. Oncogene 2006; 25: 4998-5010.

61. Easton DF, Pooley KA, Dunning AM, Pharoah PD, Thompson D, Ballinger DG et al. Genome-wide association study identifies novel breast cancer susceptibility loci. Nature 2007: 447: 1087-1093.

62. Cancer Genome Atlas NComprehensive molecular portraits of human breast tumours. Nature 2012; 490: 61-70.

63. Fanale D, Amodeo V, Corsini LR, Rizzo S, Bazan V, Russo A. Breast cancer genome-wide association studies: there is strength in numbers. Oncogene 2012; 31: 2121-2128.

64. Ellis MJ, Ding L, Shen D, Luo J, Suman VJ, Wallis JW et al. Whole-genome analysis informs breast cancer response to aromatase inhibition. Nature 2012; 486: 353-360.

65. Latif A, Hadfield KD, Roberts SA, Shenton A, Lalloo F, Black GC et al. Breast cancer susceptibility variants alter risks in familial disease. J Med Genet 2010; 47: 126-131.

66. Gorodnova TV, Kuligina E, Yanus GA, Katanugina AS, Abysheva SN, Togo AV et al. Distribution of FGFR2, TNRC9, MAP3K1, LSP1, and 8q24 alleles in genetically enriched breast cancer patients versus elderly tumor-free women. Cancer Genet Cytogenet 2010; 199: 69-72.

67. Santisteban M, Reiman JM, Asiedu MK, Behrens MD, Nassar A, Kalli KR et al. Immune-induced epithelial to mesenchymal transition in vivo generates breast cancer stem cells. Cancer Res 2009; 69: 2887-2895.

68. Ripperger T, Gadzicki D, Meindl A, Schlegelberger B. Breast cancer susceptibility: current knowledge and implications for genetic counselling. Eur J Human Genet 2009; 17: 722-731.

69. Ni TK, Landrette SF, Bjornson RD, Bosenberg MW, Xu T. Low-copy piggyBac transposon mutagenesis in mice identifies genes driving melanoma. Proc Natl Acad Sci USA 2013; 110: E3640-E3649.

70. Tan TT, Coussens LM. Humoral immunity, inflammation and cancer. Curr Opin Immunol 2007; 19: 209-216.

71. Zweifel ME, Leahy DJ, Barrick D. Structure and Notch receptor binding of the tandem WWE domain of Deltex. Structure 2005; 13: 1599-1611.

72. Charlaftis N, Suddason T, Wu X, Anwar S, Karin M, Gallagher E. The MEKK1 PHD ubiquitinates TAB1 to activate MAPKs in response to cytokines. EMBO J 2014; 33 2581-2596.

73. Liu WH, Lai MZ. Deltex regulates T-cell activation by targeted degradation of active MEKK1. Mol Cell Biol 2005; 25: 1367-1378.

74. Merbl Y, Kirschner MW. Protein microarrays for genome-wide posttranslational modification analysis. WIRE Syst Biol Med 2011; 3: 347-356.

75. Merbl Y, Kirschner MW. Large-scale detection of ubiquitination substrates using cell extracts and protein microarrays. Proc Natl Acad Sci USA 2009; 106: 2543-2548.

76. Shibuya H, Yamaguchi K, Shirakabe K, Tonegawa A, Gotoh Y, Ueno N et al. TAB1: an activator of the TAK1 MAPKKK in TGF-beta signal transduction. Science 1996; 272 : 1179-1182.
77. Kanayama A, Seth RB, Sun L, Ea CK, Hong M, Shaito A et al. TAB2 and TAB3 activate the NF-kappaB pathway through binding to polyubiquitin chains. Mol Cell 2004; 15: 535-548.

78. Wu X, Zhang W, Font-Burgada J, Palmer T, Hamil AS, Biswas SK et al. Ubiquitin-conjugating enzyme Ubc13 controls breast cancer metastasis through a TAK1-p38 MAP kinase cascade. Proc Natil Acad Sci USA 2014; 111: 13870-13875.

79. Yamashita M, Fatyol K, Jin C, Wang X, Liu Z, Zhang YE. TRAF6 mediates Smadindependent activation of JNK and p38 by TGF-beta. Mol Cell 2008; 31: 918-924.

80. Xu P, Davis RJ. C-Jun NH2-terminal kinase is required for lineage-specific differentiation but not stem cell self-renewal. Mol Cell Biol 2010; 30: 1329-1340.

81. Barruet E, Hadadeh O, Peiretti F, Renault VM, Hadjal Y, Bernot D et al. p38 mitogen activated protein kinase controls two successive-steps during the early mesodermal commitment of embryonic stem cells. Stem Cells Dev 2011; 20: 1233-1246.

82. Gaur M, Ritner C, Sievers R, Pedersen A, Prasad M, Bernstein HS et al. Timed inhibition of p38MAPK directs accelerated differentiation of human embryonic stem cells into cardiomyocytes. Cytotherapy 2010; 12: 807-817.

83. Bonnesen B, Orskov C, Rasmussen S, Holst PJ, Christensen JP, Eriksen KW et al. MEK kinase 1 activity is required for definitive erythropoiesis in the mouse fetal liver. Blood 2005; 106: 3396-3404.

84. Goto M, Chow J, Muramoto K, Chiba K, Yamamoto S, Fujita M et al. E6201 [(3S,4R,5Z,8S,9S,11E)-14-(ethylamino)-8, 9,16-trihydroxy-3,4-dimethyl-3,4,9,19-tetrahydro$1 \mathrm{H}$-2-benzoxacyclotetradecine-1,7 (8H)-dione], a novel kinase inhibitor of mitogen-activated protein kinase/extracellular signal-regulated kinase kinase (MEK)-1 and MEK kinase-1: in vitro characterization of its anti-inflammatory and antihyperproliferative activities. J Pharmacol Exp Therapeut 2009; 331: 485-495.

85. Su YC, Han J, Xu S, Cobb M, Skolnik EY. NIK is a new Ste20-related kinase that binds NCK and MEKK1 and activates the SAPK/JNK cascade via a conserved regulatory domain. EMBO J 1997; 16: 1279-1290.

86. Fanger GR, Widmann C, Porter AC, Sather S, Johnson GL, Vaillancourt RR. 14-3-3 proteins interact with specific MEK kinases. J Biol Chem 1998; 273: 3476-3483.

87. Xu S, Cobb MH. MEKK1 binds directly to the c-Jun N-terminal kinases/stress-activated protein kinases. J Biol Chem 1997; 272: 32056-32060.

88. Karandikar M, Xu S, Cobb MH. MEKK1 binds raf-1 and the ERK2 cascade components. J Biol Chem 2000; 275: 40120-40127.

89. Gallagher ED, Gutowski S, Sternweis PC, Cobb MH. RhoA binds to the amino terminus of MEKK1 and regulates its kinase activity. J Biol Chem 2004; 279: 1872-1877.

90. Fanger GR, Johnson NL, Johnson GL. MEK kinases are regulated by EGF and selectively interact with Rac/Cdc42. EMBO J 1997; 16: 4961-4972.

91. Russell M, Lange-Carter CA, Johnson GL. Direct interaction between Ras and the kinase domain of mitogen-activated protein kinase kinase kinase (MEKK1). J Biol Chem 1995; 270: 11757-11760.

92. Christerson LB, Gallagher E, Vanderbilt CA, Whitehurst AW, Wells C, Kazempour R et al. p115 Rho GTPase activating protein interacts with MEKK1. J Cell Physiol 2002; 192: 200-208.

93. Baud V, Liu ZG, Bennett B, Suzuki N, Xia Y, Karin M. Signaling by proinflammatory cytokines: oligomerization of TRAF2 and TRAF6 is sufficient for JNK and IKK activation and target gene induction via an amino-terminal effector domain. Genes Dev 1999; 13 1297-1308

94. Pomerance M, Multon MC, Parker F, Venot C, Blondeau JP, Tocque B et al. Grb2 interaction with MEK-kinase 1 is involved in regulation of Jun-kinase activities in response to epidermal growth factor. J Biol Chem 1998; 273: 24301-24304.

95. Zhang Y, Neo SY, Wang X, Han J, Lin SC. Axin forms a complex with MEKK1 and activates c-Jun $\mathrm{NH}(2)$-terminal kinase/stress-activated protein kinase through domains distinct from Wnt signaling. J Biol Chem 1999; 274: 35247-35254.

96. Yujiri T, Nawata R, Takahashi T, Sato Y, Tanizawa Y, Kitamura T et al. MEK kinase 1 interacts with focal adhesion kinase and regulates insulin receptor substrate-1 expression. J Biol Chem 2003; 278: 3846-3851.

97. Yin MJ, Christerson LB, Yamamoto Y, Kwak YT, Xu S, Mercurio F et al. HTLV-I Tax protein binds to MEKK1 to stimulate IkappaB kinase activity and NF-kappaB activation. Cell 1998; 93: 875-884.

98. Ritterhoff S, Farah CM, Grabitzki J, Lochnit G, Skurat AV, Schmitz ML. The WD40-repeat protein Han11 functions as a scaffold protein to control HIPK2 and MEKK1 kinase functions. EMBO J 2010; 29: 3750-3761.

99. Pearlman A, Loke J, Le Caignec C, White S, Chin L, Friedman A et al. Mutations in MAP3K1 cause $46, X Y$ disorders of sex development and implicate a common signal transduction pathway in human testis determination. Am J Human Genet 2010; 87: 898-904.

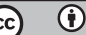

This work is licensed under a Creative Commons Attribution 3.0 Unported License. The images or other third party material in this article are included in the article's Creative Commons license, unless indicated otherwise in the credit line; if the material is not included under the Creative Commons license, users will need to obtain permission from the license holder to reproduce the material. To view a copy of this license, visit http://creativecommons.org/ licenses/by/3.0/ 